\title{
Compliant Ultrasound Proximity Sensor for the Safe Operation of Human Friendly Robots Integrated with Tactile Sensing Capability
}

\author{
Il-Joo Cho*, Hyung-Kew Lee ${ }^{\dagger}$, Sun-Il Chang** and Euisik Yoon***
}

\begin{abstract}
The robot proximity and tactile sensors can be categorized into two groups: grip sensors and safety sensors. They have different performance requirements. The safety sensor should have long proximity range and fast response in order to secure enough response time before colliding with ambient objects. As for the tactile sensing function, the safety sensor need to be fast and compliant to mitigate the impact from a collision. In order to meet these requirements, we proposed and demonstrated a compliant integrated safety sensor suitable to human-friendly robots. An ultrasonic proximity sensor and a piezoelectric tactile sensor made of PVDF films have been integrated in a compliant PDMS structure. The implemented sensor demonstrated the maximum proximity range of $35 \mathrm{~cm}$. The directional tolerance for $30 \mathrm{~cm}$ detection range was about $\pm 15^{\circ}$ from the normal axis. The integrated PVDF tactile sensor was able to detect various impacts of up to $20 \mathrm{~N}$ in a controlled experimental setup.
\end{abstract}

Keywords: Tactile sensor, Proximity sensor, Ultrasonic sensor, Human-friendly robots, PDMS

\section{Introduction}

In recent years, interests in robot research have been moving towards human-friendly robots. Human-friendly robots are assumed to share the same workspace and interact with humans. Therefore, safety of interaction between them is as important as performance of the robots is [1]. The performance of the human-friendly robots often means the performance of gripping target objects. On the other hand, the safety of the interaction mostly means avoiding collisions between robots in action and surrounding objects and minimizing the impact if a collision occurs. The major sensor systems for the humanrobot interaction except vision systems are proximity and tactile sensors. Hence, various proximity sensors [2-6] and tactile sensors [7-12] have been reported for either the grip performance or the safety of interaction.

In recent years, integrated sensors functioning as both proximity and tactile sensors have been explored as an effort to save cost and space [13-16]. Göger et al reported a capacitive tactile sensor module that is capable of sensing proximity in combination with another module in the different operational mode [13, 14]. They employed a polymer foam between electrode layers as a deformable dielectric to sense the tactile pressure. The top electrode of their sensor operates in two different modes: generating or receiving electromagnetic waves. The proximity of objects can be detected by electric fields between modules in

$\dagger$ Corresponding Author: Center for Electricity and Magnetism, KRISS, Korea. (hyungkew.lee@kriss.re.kr)

* Center for Biosystems, KIST, Korea. (ijcho@kist.re.kr)

** University of Michigan, USA. (sunil.chang@gmail.com)

*** Dept. of EECS, University of Michigan, USA. (esyoon@umich.edu)

Received: August 19, 2016; Accepted: October 22, 2016 different modes. In another work, Konstantinova et al employed fiber optics to implement both tactile and proximity sensors in a fingertip of a robot [15]. A lightemitting fiber tip and a receiving fiber tip were installed to face an elastomer layer which makes a contact with an object. The intensity of the reflected light from a surface of the elastomer provides the contact information. Another set of fiber tips without a soft reflector were used for the proximity sensing. The light reflected directly from the approaching object is detected through a receiving fiber.

Our group also has reported an integrated sensor that is small and thin enough to be installed on a robot finger [16]. A 16 by 16 capacitive tactile sensor array with 1 $\mathrm{mm}$ special resolution was realized in a $1 \mathrm{~mm}$ thick polydimethylsiloxane (PDMS) sheet. It measures the proximity by taking advantage of the top electrode array deployed for the tactile sensors. However, our previous sensor was not adequate for the safety sensor because of the relatively short proximity range and the susceptibility to the characteristics of approaching objects. Therefore, in this work, we devised a new compliant ultrasonic proximity sensor to achieve longer proximity range over 30 $\mathrm{cm}$ with less susceptibility to the characteristics of the objects integrated with a tactile sensing capability, which is suitable to the safe interaction between humans and robots.

\section{Sensor Design and Implementation}

\subsection{Sensor design}

Fig. 1 describes a scenario of a robot arm which is about to grip an object of an irregular shape while avoiding 


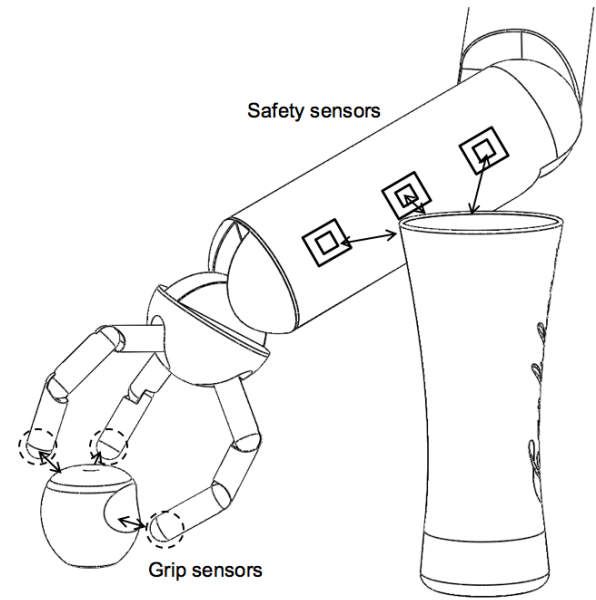

Fig. 1. A robot arm gripping an irregular object while avoiding collision with the cup by using safety sensors

collision with a tall cup as an ambient object. Two kinds of sensors are involved in this scenario. One is the grip sensor for hands and the other is the safety sensor for arms. Both sensors should have proximity and tactile sensing functions. The role of the grip sensor in proximity mode is to make the robot fingers touch the irregular surface simultaneously for a stable grip by keeping the distances between the fingers and the surface same as described in [5]. The tactile sensing function of the grip sensor provides contact force to enable fine manipulation of objects. The role of the safety sensor is to provide distance information to the cup quickly in order for the robot to avoid a collision in the proximity mode and the contact force to reduce the impact from the collision in the tactile mode when it occurs. The grip sensors are mostly installed in the fingers while the safety sensors are usually placed in the arms as depicted in the figure.

The requirements for each sensor in the scenario are also different. The requirements for the grip sensor are small size and high resolution for both proximity and tactile sensing modes. However, the requirements for the safety sensors in proximity mode are long range and fast response in order to secure enough response time before a collision with ambient objects. The response also should not be affected severely by the size and the material of approaching objects. In tactile mode, the safety sensor should be fast and compliant to mitigate the potential damage from impact. It does not need to have high special resolution as the grip sensor does.

The performance of our previous sensor does not meet the requirements for the safety sensor [16]. Therefore, we proposed a compliant safety sensor design to meet those requirements in this work. The sensor is made of PDMS with embedded polyvinylidene fluoride (PVDF) films to form an ultrasonic proximity sensor and a piezoelectric tactile sensor together in a single module. The ultrasonic proximity sensors are able to detect an approaching object

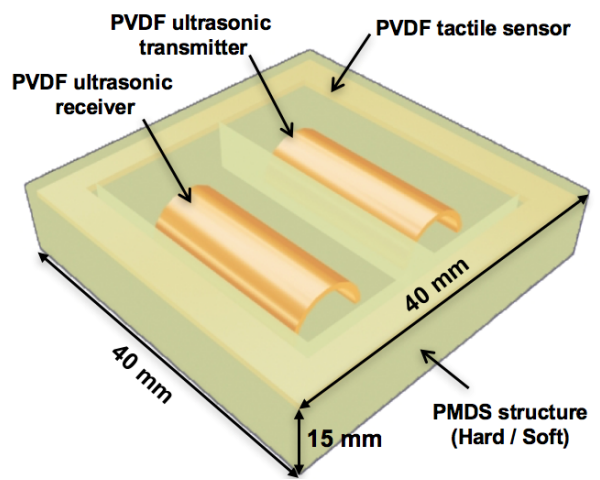

(a)

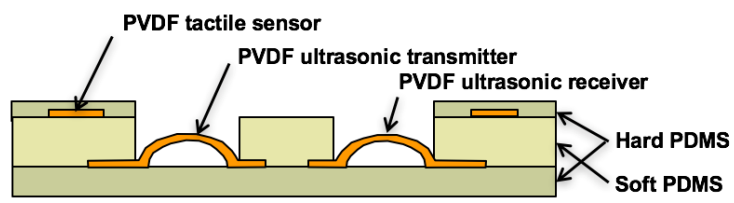

(b)

Fig. 2. (a) The three dimensional sensor structure and (b) its cross-section

at a few tens of centimeters and fast enough to secure some time for a robot to respond. The piezoelectric tactile sensors also have fast response owing to its capacitive nature. The sensor design is illustrated in Fig. 2. It consists of three PDMS and three PVDF layers with electrodes coated on both sides. Two PVDF layers deployed in a cylindrical shape on a hard PDMS layer work as an ultrasonic transmitter and a receiver, respectively. We separated the transmitter and the receiver not to lose the reflected sound from close objects. The distance between the transmitter and the receiver is $6 \mathrm{~mm}$. The window for the soundwave is $10 \mathrm{~mm}$ by $25 \mathrm{~mm}$. The top PVDF layer is deployed on top of the soft PDMS layer functioning as a tactile sensor. When a collision occurs, the soft PDMS deforms and the PVDF tactile sensor produces electric charges due to the piezoelectricity of the film.

\subsection{Sensor fabrication and system implementation}

We used a $26 \mu$ m-thick commercial PVDF film from Precision Acoustics, UK. It has piezoelectric coefficients of $-6 \mathrm{pC} / \mathrm{N}$ at $\mathrm{d}_{31}$ and $-32 \mathrm{pC} / \mathrm{N}$ at $\mathrm{d}_{33}$. The PDMS layers were fabricated by mixing a liquid PDMS from Dow Corning, CA, USA with a curing agent and curing them in a mold. The hardness of the cured layer was controlled by the mixing ratio. The mixing ratios for the hard and soft PDMS layers were 1:2 and 1:10 in weight, respectively. The PDMS layers were bonded chemically with PVDF layers embedded in between after oxygen plasma treatment of PDMS surfaces. Fig. 3(a) shows the fabricated proximity sensor. Three pairs of interconnection lines dedicated for the transmitter, the receiver, and the tactile sensor run out of the fabricated sensor. 


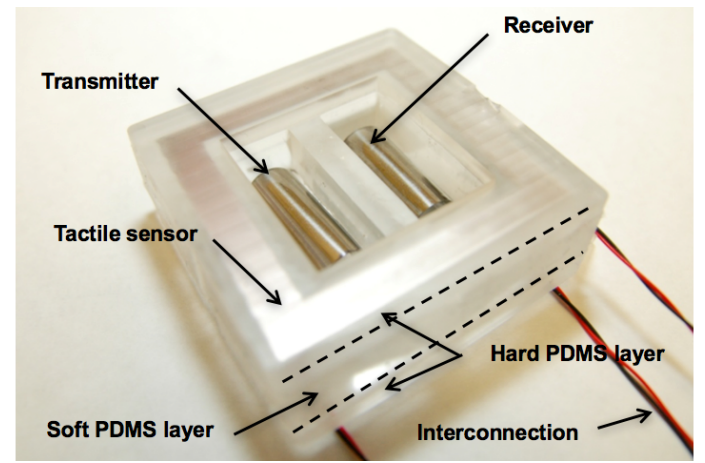

(a)

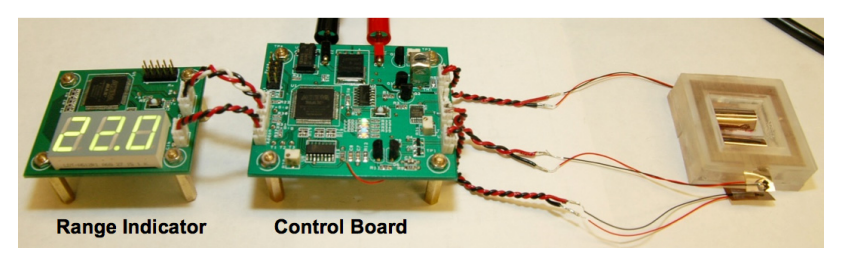

(b)

Fig. 3. (a) The fabricated piezoelectric safety sensor using PVDF films and PDMS structure and (b) the control system

Fig. 3(b) displays a photograph of the implemented system. The control board in the figure receives a trigger signal from the robot central system, drives the ultrasonic transmitter, detect the reflected ultrasound, and generates timing signal with which the distance of the object is calculated. The range indicator emulates the robot central system and display the calculated distance to the detected object. The control board also amplifies the tactile signal from the contact and transfer it to the robot central system.

\subsection{System operation}

Fig. 4 describes the proximity and tactile operation of the control board. The field programmable gate array (FPGA) chip in Fig. 4(a) has been programmed to conduct all the required digital functions such as timing, computation, and comparison. When the robot central system issues a trigger signal to the board, the FPGA chip generates a burst of five pulses separated by $25 \mu \mathrm{sec}$ which corresponds to $40 \mathrm{kHz}$ frequency. Then, the high voltage circuit amplifies the pulses to $\pm 50 \mathrm{~V}$ to drive the PVDF transmitter. The reflected soundwave is converted to the voltage signal through the receiver, amplified by a low noise amplifier (LNA), and then compared with the internal threshold level after converted to a digital signal by an analog to digital converter (ADC). If the reflected signal is larger than the internal threshold, the FPGA generates a timing signal with which the robot central system calculates the distance to the detected object. The update rate of the proximity information is $10 \mathrm{msec}$. The resolution of the proximity sensing which is determined by the sampling rate $(1 \mathrm{MHz})$ of the ADC is $0.17 \mathrm{~mm}$.

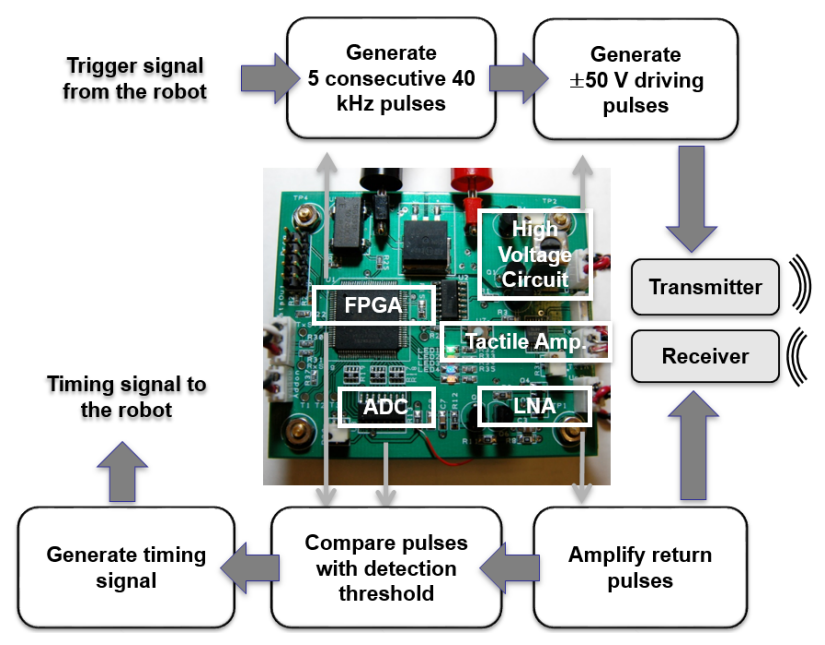

(a)

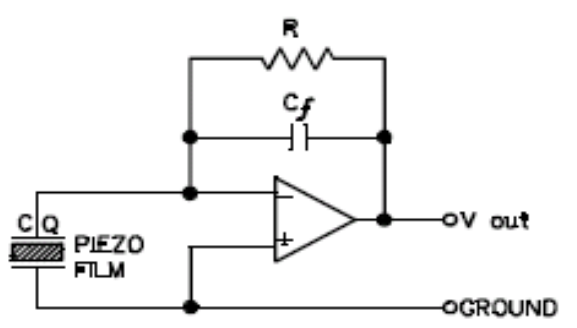

(b)

Fig. 4. (a) Functional blocks in the implemented control board and the operation for the proximity detection; (b) the schematic of the tactile amplifier in the control board

The operation of the tactile sensor is much simpler than the proximity sensor because it does not require timing function. A tactile amplifier shown in Fig. 4(b) monitors the voltage across the PVDF tactile sensor. When an impact occurs, the PVDF tactile sensor deforms and a voltage appears across the film due to the change of surface charges. The voltage is amplified by the ratio of the feedback capacitance to the capacitance of the PVDF film $\left(-\mathrm{C} / \mathrm{C}_{f}\right)$ to produce the output voltage. Since the stored charges in $\mathrm{C}_{f}$ dissipate through the feedback resistor $\mathrm{R}$, the tactile sensor cannot keep the output voltage from a static pressure. This is a typical characteristic of piezoelectric sensors. Notwithstanding, this is not an issue for the safety sensor application to detect a collision because it is not a static event. Actually, this characteristic even resembles that of human skin. The values of feedback components were $\mathrm{R}=10 \mathrm{k} \Omega$ and $\mathrm{Cf}=100 \mathrm{nF}$, respectively. The update rate of the tactile information is $10 \mathrm{msec}$.

\section{Experimental Results and Discussions}

\subsection{Proximity detection}

Fig. 5 shows the amplified waveform from the receiver 
and the timing signal generated by comparison between the waveform amplitude and the preset threshold in a single screen. The full horizontal scale is $2 \mathrm{msec}$. The full vertical scale for the reflected signal and the timing signal are 800 $\mathrm{mV}$ and $8 \mathrm{~V}$, respectively. Fig. 5(a) displays a typical signal pattern produced by the receiver. Large pulses were observed at the beginning of the acquisition due to the interference from the transmitter. This is because the transmitter and the receiver are deployed in a close position and connected mechanically through PDMS layers as shown in Fig. 2. Other small interferences were also observed throughout the acquisition. They are likely to be caused by the multiple interference paths between the

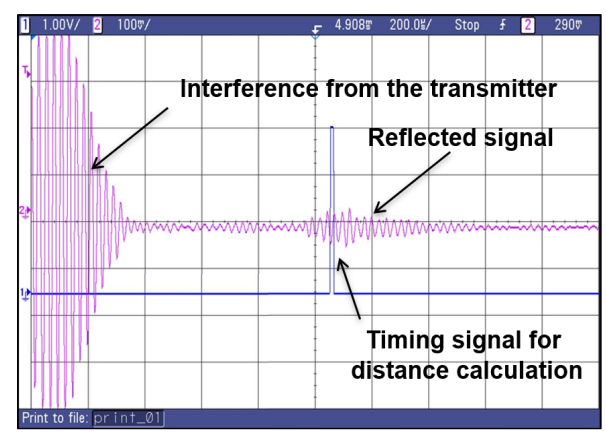

(a)

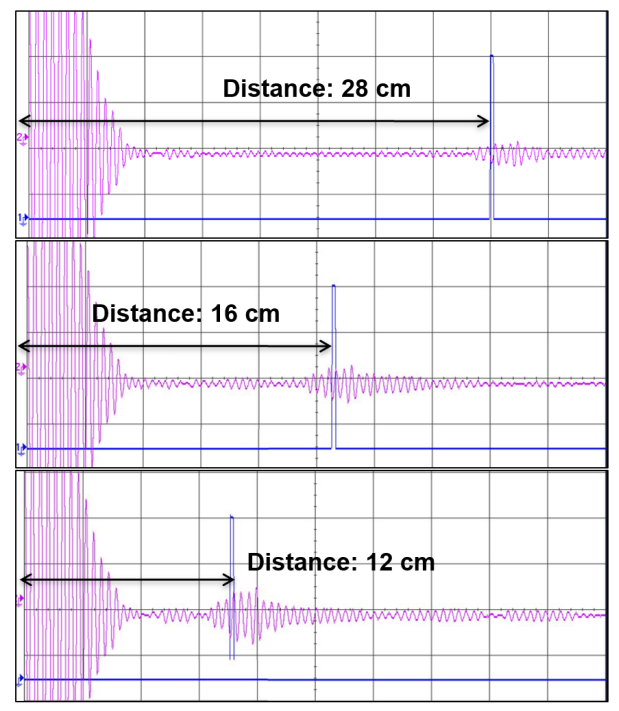

(b)

Fig. 5. (a) A signal waveform from the receiver after LNA displayed with the timing signal and (b) signals with varying distance to the approaching object transmitter and the receiver. We also found that the reflected waveform is longer than expected. Since the driving signal had five pulses, the reflected signal should have the same number of pulses. However, it showed more than five pulses as noticed in Fig. 5. It is probable that the soundwave emitted from the backside (concave side) of the transmitter was reflected at the hard PDMS layer and then superposed on the wave emitted from the front side (convex side). In order to solve the problems mentioned above, we need to adopt an acoustic insulator and optimize the design of the PDMS structure through sophisticated acoustic simulations. These will be our next step of the development.

The maximum and minimum detection range were $35 \mathrm{~cm}$ and $5 \mathrm{~cm}$ at normal direction to the surface, respectively. The maximum detection range is determined by the magnitude of unwanted interferences mentioned earlier. The minimum detection range was limited to $5 \mathrm{~cm}$ due to the large interference from the transmitter at the beginning of the acquisition. Fig. 6 shows the maximum detection range with respect to the approaching direction of an object. The directional tolerance for $30 \mathrm{~cm}$ detection range was about $\pm 15^{\circ}$.

We tested different objects such as a metal, a plastic, a bare hand, and a hand with a woolen glove. The measured distance of the tested objects did not show significant difference among the objects. On the other hand, the magnitude of reflected signal was affected by the softness of the material. Therefore, the maximum detection range for each material is different. Even though there is some susceptibility to the softness, the maximum detection range was over $35 \mathrm{~cm}$. We summarized the characteristics of the proximity sensors integrated with the tactile sensing function for robot applications in Table 1.

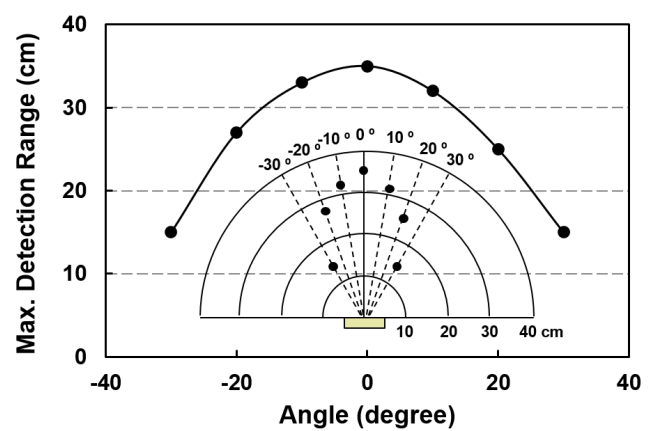

Fig. 6. Maximum detection range with respect to the angles from the normal axis to the sensor

Table 1. Characteristics of the proximity sensors integrated with tactile sensing functions for robot applications

\begin{tabular}{|c|c|c|c|c|c|}
\hline References & Sensing method & $\begin{array}{l}\text { Max. detection } \\
\text { range }\end{array}$ & Sensor area & $\begin{array}{c}\text { Flexibility / } \\
\text { Compliant surface }\end{array}$ & $\begin{array}{c}\text { Susceptibility to the } \\
\text { characteristics of objects }\end{array}$ \\
\hline$[13],[14]$ & Capacitive & $30 \mathrm{~cm}$ (hand) & $40 \times 40 \mathrm{~mm}^{2}$ & $\mathrm{~N} / \mathrm{Y}$ & High \\
\hline$[15]$ & Optical & $2 \mathrm{~cm}$ & $16.5 \times 16.5 \mathrm{~mm}^{2}$ & $\mathrm{~N} / \mathrm{N}$ & High \\
\hline$[16]$ & Capacitive & $17 \mathrm{~cm}$ (hand) & $22 \times 22 \mathrm{~mm}^{2}$ & $\mathrm{Y} / \mathrm{Y}$ & High \\
\hline This work & Ultrasonic (time of flight) & $35 \mathrm{~cm}$ (hand) & $40 \times 40 \mathrm{~mm}^{2}$ & $\mathrm{Y} / \mathrm{Y}$ & Low \\
\hline
\end{tabular}



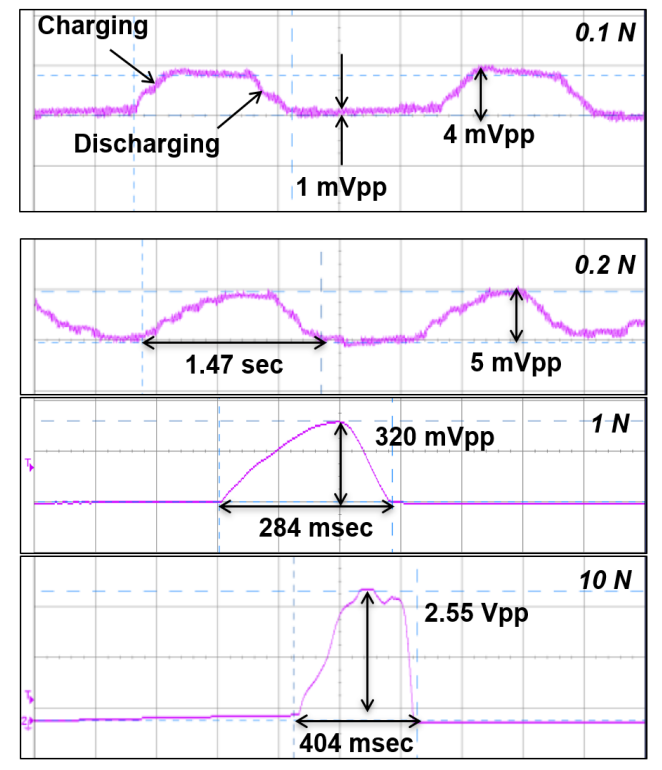

Fig. 7. Responses of the PVDF tactile sensor with respect to the various impact forces

\subsection{Tactile sensing}

The tactile sensor was tested in a controlled situation where simulated impacts were applied by a motorized zstage with a force gauge attached to it, which has been used for this project [7]. The output responses of the tactile sensor from various impacts shown in Fig. 7 were monitored with an oscilloscope while a $10 \mathrm{~mm}$ diameter tip of the force gauge was pushing and releasing the surface of the sensor body. The noise floor of the amplified output was $1 \mathrm{mVpp}$. Forces from $0.1 \mathrm{~N}$ up to $20 \mathrm{~N}$ were applied to the surface of the sensor with the tip. In a real situation, it is difficult to infer an applied force from a colliding object quantitatively unless the detailed information of the contact surface is known in our current design. This problem will be solved by implementing an array of tactile sensors with the formation of multiple electrodes on one surface of the PVDF film in the next stage of the development.

\subsection{Discussions for further improvements}

As we mentioned in the previous sections, the design of our first prototype requires improvements. The design should be optimized to minimize the interference from the transmitter to the receiver. In addition to this, the two PVDF layers can be integrated into one by patterning multiple electrodes on a PVDF films to reduce the thickness of the whole structure. The sensitivity of tactile sensing can also be improved by forming PDMS layers with micro structures. These improvements will be made in our second round of the research. We are also planning to equip a setup to test the dynamic response of the tactile sensing mode of our sensor.

\section{Conclusions}

In this report, we proposed and demonstrated our first prototype of the compliant integrated safety sensor designed for robot applications. An ultrasonic proximity sensor and piezoelectric tactile sensor were implemented in a compliant PDMS body with PVDF films embedded. The sensor demonstrated the maximum proximity range of 35 $\mathrm{cm}$. The directional tolerance assuring $30 \mathrm{~cm}$ detection range was about $\pm 15^{\circ}$ from the normal axis. The integrated PVDF tactile sensor could detect the various impacts induced by an object in a controlled experimental setup. Through further improvements, the proposed sensor could contribute to the realization of safe human friendly robots in the future as a safety sensor.

\section{Acknowledgements}

This work was supported by Brain Pool Program through the Korean Federation of Science and Technology Societies (KOFST) funded by the Ministry of Science, ICT and Future Planning

\section{References}

[1] Zinn, M., Roth, B., Khatib, O., and Slisbury, J., "A New Actuation Approach for Human Friendly Robot Design,” Int. J. Robot. Res. Vol. 23, No. 4-5, pp. 379398, 2004.

[2] Novak, J. L., and Feddema, I. T., "A capacitive-based proximity sensor for whole arm obstacle avoidance," IEEE Int. Conf. Robot. Autom., pp. 1307-1314, 1992.

[3] Volpe, R. and Ivlev, R., "A survey and experimental evaluation of proximity sensors for space robots," IEEE Int. Conf. Robot. Autom., pp. 3466-3473, 1994.

[4] Bonen, A., Saad, R. E., Smith, K. C., and Benhabib, B., "A novel electrooptical proximity sensor for robotics: Calibration and active sensing," IEEE Trans. Robot. Autom., Vol. 13, No. 3, pp. 377-386, 1997.

[5] Smith, J., Garcia, E., Wistort, R., and Krishinamoorthy, G., "Electric Field Imaging Pretouch for Robotic Graspers," in IEEE/RJS International Conference on Intelligent Robots and Systems, pp. 676683, 2007.

[6] Mayton, B., LeGrand, L., and Smith, J., "An Electric Field Pretouch System for Grasping and Co-Manipulation,” IEEE Int. Conf. Robot. Autom., pp. 831838, 2010.

[7] Lee, H.-K., Chang, S.-I., and Yoon, E., "A flexible polymer tactile sensor: fabrication and modular expandability for large area deployment," J. Microelectromech. Syst. Vol. 15, No. 6, pp. 1681-1686, 2006. 
[8] Lee, H.-K., Chang, S.-I., and Yoon, E., "Normal and shear force measurement using a flexible polymer tactile sensor with embedded multiple capacitors," J. Microelectromech. Syst. Vol. 17, No. 4, pp. 934-942, 2008.

[9] Lee, H.-K., Chung, J., Chang, S.-I., and Yoon, E., "Real-time measurement of the three-axis contact force distribution using a flexible capacitive polymer tactile sensor," J. Micromech. Microeng. Vol. 21, No. 3, 035010, 2011.

[10] Ulmen, J. and Cutkosky, M., "A Robust, Low-Cost and Low-Noise Artificial Skin for Human-Friendly Robots," IEEE Int. Conf. Robot. Autom., pp. 48364841, 2010.

[11] Phan, S., Quek, Z. F., Shah, P., Shin, D., Ahmed, Z., Khatib, O., and Cutkosky, M., "Capacitive Skin Sensors for Robot Impact Monitoring," in IEEE/RSJ International Conference on Intelligent Robots and Systems, pp. 2992-2997, 2011.

[12] Charalambides, A. and Bergbreiter, S., "A novel allelastomer MEMS tactile sensor for high dynamic range shear and normal force sensing," J. Micromech. Microeng. Vol. 25, No. 9, 095009, 2015.

[13] Goeger, D., Blanketz, M., and Woern, H., “A Tactile Proximity Sensor,” in IEEE Sensors Conference, pp. 589-594, 2010.

[14] Göger, D., Alagi, H., and Wörn, H., "Tactile proximity sensors for robotic applications," in IEEE International Conference on Industrial Technology, pp. 978-983, 2013.

[15] Konstantinova, J., Stilli, A., and Althoefer, K., "Force and proximity fingertip sensor to enhance grasping perception," in IEEE/RSJ International Conference on Intelligent Robots and Systems, pp. 2118-2123, 2015.

[16] Lee, H.-K., Chung, J., Chang, S.-I., and Yoon, E., "Dual-Mode Capacitive Proximity Sensor for Robot Application: Implementation of Tactile and Proximity Sensing Capability on a Single Polymer Platform Using Shared Electrodes," IEEE Sensors J. Vol. 9, No. 12, pp. 1748-1755, 2009.

Il-Joo Cho He received the B.S., M.S. and Ph.D. degrees in electrical engineering from Korea Institute of Science and Technology (KAIST), Daejeon, Korea in 1998, 2000and 2004, respectively. From 2004 to 2007 he was with LG Electronics Institute of Technology, Seoul, where he was engaged in researches on RF MEMS switch and nano data storage system. From 2007 to 2008, he was a research fellow at University of Minnesota, Minneapolis, MN, working on tactile sensors. From 2008 to 2010, he was a visiting research scientist at University of Michigan, Ann Arbor, MI, where he was engaged in MEMS neural probe. In 2010, he joined Center for BioMicrosystems at
Korea Institute of Science and Technology (KIST), Seoul, Korea, where he is now an senior research scientist. His present research interests are in MEMS neural probe, Lab on a chip, implant able microsystems. Currently, he is serving as a executive technical program committee member for the IEEE International conference on Micro Electro Mechanical Systems2015

Hyung-Kew Lee He received his BS degree in 1996 and MS degree in 1999 in Electrical Engineering from the Korea Advanced Institute of Science and Technology (KAIST). He worked on uncooled infrared bolometers for his Master thesis. He received his $\mathrm{PhD}$ degree from KAIST in 2004 for the research about a tunable optical filter. He joined the University of Minnesota in 2005 as a postdoctoral research scholar and worked on tactile devices for robots and mobile handsets until 2007. He joined Samsung Electronics at 2010 for haptic interface research. Since 2012, he is working at Korea Institute of Standards and Science as a senior scientist. His primary research interests are tactile interface system, flexible electronics, microsystems, and photonics.

Sun-Il Chang He received the B.S. degree in electrical engineering from Korea University, Seoul, Korea, in 2003. $\mathrm{He}$ received his $\mathrm{Ph} . \mathrm{D}$. degree in the Department of Electrical Engineering and Computer Science, University of Michigan, Ann Arbor. In 2013, He joined Marvell Semiconductor, Inc.

Euisik Yoon He received the B.S. and M.S. degrees in electronics engineering from Seoul National University, Seoul, Korea, in 1982 and 1984, respectively, and the Ph.D. degree in electrical engineering from the University of Michigan, Ann Arbor, in 1990. From 1990 to 1994, he was with the Fairchild Research Center, National Semiconductor Corporation. Santa Clara, CA, where he was engaged in researching deep-submicrometer CMOS integration and advanced gate dielectrics. From 1994 to 1996, he was a Member of Technical Staff with Silicon Graphics Inc., Mountain View, CA, where he worked on the design of the MIPS microprocessor R4300i and the RCP 3-D graphic coprocessor. From 1996 to 2005, he was with the Department of Electrical Engineering, Korea Advanced Institute of Science and Technology (KAIST), Daejeon, Korea. From 2005 to 2008, he was with the Department of Electrical and Computer Engineering, University of Minnesota, Minneapolis. During the academic year of 2000-2001, he was a Visiting Faculty at Agilent Laboratory, Palo Alto, CA. In 2008, he joined the Department of Electrical Engineering and Computer Science, University of Michigan, Ann Arbor, MI. His research interests are MEMS, integrated microsystems, and 
VLSI circuit design. Dr. Yoon was a corecipient of the 1999 and 2000 Student Paper Award at the IEEE International Microwave Symposium, concerning the topics on MEMS inductors and RF MEMS switch work. He has served on various Technical Program Committees, including those of the Microprocesses and Nanotechnology Conference, the International Sensor Conference, the IEEE Asia-Pacific Conference on Advanced System Integrated Circuits, the IEEE

International Conference on Micro Electro Mechanical Systems, and the Transducers Conference. He also served on the IEEE International Solid-State Circuit Conference Program Committee and is currently serving on the IEEE International Electron Devices Meeting Executive Committee. 